\title{
Manutenção térmica em galinhas domésticas mantidas sob anestesia inalatória com isofluorano
}

\author{
Thermal maintenance in chickens under inhalant anesthesia with isoflurane
}

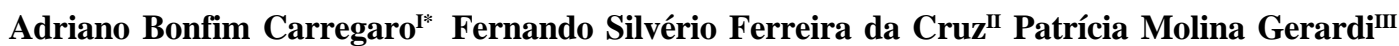 \\ Claudia Scholten ${ }^{\mathrm{III}}$
}

\section{RESUMO}

A hipotermia em aves sob anestesia é freqüentemente observada, causando alterações fisiológicas e metabólicas que podem levar ao óbito. O estudo avaliou dois métodos de manutenção térmica em galinhas submetidas à anestesia inalatória com isofluorano. Foram utilizadas seis galinhas, pesando entre 1 e 1,5kg, com idade aproximada de um ano. Os animais foram autocontrole, submetidos a três grupos: controle (GC), não sendo utilizado nada; alumínio (GA), envoltos por papel alumínio comercial; manta (GM) envoltos por uma manta térmica de dupla face de alumínio. Após jejum prévio de quatro horas, as aves foram submetidas à anestesia inalatória por meio de máscara facial com $4 \mathrm{~V} \%$ de isofluorano e mantidas posteriormente com $2 \mathrm{~V} \%$ do mesmo agente inalatório, em um circuito avalvular com umidificador, após intubação endotraqueal. As aves foram mantidas em sala climatizada $\left(26^{\circ} \mathrm{C}\right)$ e posicionadas em decúbito lateral. As variáveis mensuradas foram a temperatura corpórea, freqüência cardíaca, freqüência respiratória e saturação de oxihemoglobina, nos momentos zero, 5, 15, 30, 45, 60, 75 e 90 minutos. Tanto no GC quando no GM houve hipotermia aos 15 minutos, acentuando-se até o fim das mensurações. No GA a hipotermia só ocorreu após 45 minutos. Houve bradicardia no GC a partir de 15 minutos, no GM a partir de 30 minutos e no GA somente após 60 minutos. Observou-se bradipnéia no GC após 45 minutos e aos 15 e 30 minutos no GA e GM, respectivamente. Ambos os métodos foram eficientes na manutenção térmica, sendo o alumínio mais eficiente devido a menor variação de parâmetros fisiológicos.

Palavras-chave: termorregulação, anestesia, galinhas, Gallus gallus domesticus, isofluorano.

\section{ABSTRACT}

Hypothermia in birds under general anesthesia is frequently seen, causing physiologic and metabolic alterations that may lead to death. This study evaluated two thermic maintenance methods in chickens under inhalant anesthesia by isoflurane. Six chickens, weighing between 1 and $1.5 \mathrm{~kg}$ and with approximately one year old, were submitted to three groups in a random order: CG, control, nothing was used; GA, wrapped in a commercial aluminum paper, and GM, wrapped in a double face blanket of aluminum. After four hour fasting, they were submitted to inhalant anesthesia by facial mask with $4 \mathrm{~V} \%$ of isoflurane, and maintained with $2 \mathrm{~V} \%$ of this gas through a humidifier nonrebreathing circuit after endotracheal intubation. In GM and GA the animals body were wrapped in their totality, exception of the head. Body temperature, heart rate, respiratory rate and oxyhemoglobin saturation were measured at 0,15 , 30, 45, 60, 75 and 90 minutes. In GC and GM hypothermia occur with 15 minutes, staying in constant fall until 90 minutes. The difference in GA with basal level occurs only between 45 to 90 minutes. Bradycardia occurred in control group at 15 minutes, in blanket group at 30 minutes and in aluminum group only at 60 minutes. Bradypnea was observed in GC at 45 minutes, and at 15 and 30 minutes in GA and GM, respectively. No significant alterations were seen for to oxihemoglobin saturation. Both methods employed were effective on thermic maintenance, showing the aluminum more effective due less variation observed in body temperature and heart rate.

Key words: thermoregulation, anesthesia, chicken, Gallus gallus domesticus, isoflurane.

\section{INTRODUÇÃO}

As aves estão cada vez mais presentes nas clínicas veterinárias como animais de companhia. Com isso, procedimentos cirúrgicos ou diagnósticos em que se faz necessário o uso da contenção química ou

'Departamento de Clínica de Pequenos Animais, Centro de Ciências Rurais (CCR), Universidade Federal de Santa Maria (UFSM), 97105-900, Santa Maria, RS, Brasil. E-mail: carregaro@smail.ufsm.br. Autor para correspondência.

"Programa de Pós-graduação em Farmacologia, Centro de Ciências da Saúde (CCS), UFSM, Santa Maria, RS, Brasil.

IIIAutônomo. 
anestesia envolvendo esta classe tem se expandido rapidamente. O uso de anestésicos injetáveis em aves pode ser útil quando a anestesia inalatória não está disponível. Todavia, os agentes inalatórios são mais seguros e permitem melhor controle do plano anestésico e das alterações fisiológicas, além de produzirem rápida recuperação (PASCOE et al., 1985; CURRO et al., 1994; OLKOWSKI \& CLASSEN, 1998).

$\mathrm{Na}$ anestesia inalatória em aves, o halotano foi o agente inalatório mais empregado (LUDDERS et al., 1988; GREENLESS et al., 1990), mas vários óbitos foram associados à utilização deste agente (PASCOE et al., 1985) e o isofluorano tem se mostrado um agente seguro para a utilização em aves, produzindo menor depressão hemodinâmica que o halotano (GUNKEL \& LAFORTUNE, 2005). Foram observadas alterações no sistema hemodinâmico com a utilização do halotano e isofluorano em patos, como taquicardia acentuada e hipotensão com ambos agentes, sendo esta última maior com o uso do halotano (GOELZ et al., 1990). Ademais, a esse agente, imputou-se a ocorrência de arritmias, incluindo contrações ventriculares prematuras e taquicardias ventriculares (GOELZ et al., 1990; LUDDERS, 1992; NAGANOBU et al., 2001).

A hipotermia é freqüentemente observada durante a anestesia e em pacientes com injúria associada, podendo ocorrer acidose metabólica e distúrbios da coagulação (SMITH \& YAMAT, 2000). As causas da hipotermia consistem basicamente na redução da produção de calor pelo animal associada ao aumento da perda de calor (WATERMAN, 1981). Em anestesia inalatória, a perda de calor pela via pulmonar é elevada quando o animal inspira gás frio e seco por meio de sistemas não-reinalatórios, apesar de serem os de eleição para esses animais (FLECKNELL, 1996). Embora essas perdas possam ser reduzidas com o uso de sistemas reinalatórios, o uso destes circuitos oferece maior resistência mecânica e espaço morto para a respiração desses animais. A utilização de um umidificador pode ser eficiente para reduzir a perda de calor (DODMAN \& BRITO-BABAPULLE, 1979 apud HALL et al., 2001).

Os principais efeitos da hipotermia são a severa depressão do sistema nervoso central e de órgãos vitais. Em adição, ela pode causar danos ao sistema cardiovascular, a pele, aos órgãos internos e ao metabolismo. Durante o período de hipotermia ocorre hipotensão devido à redução do débito cardíaco, embora a resistência vascular aumente. A bradicardia pode ser devido à depressão do nodo átrio-ventricular e do feixe de His (THURMON et al., 1996).

Embora a hipotermia diminua a função metabólica e seja neuroprotetora, ela resulta em efeitos deletérios como coagulopatias, acidose metabólica e resposta imunológica suprimida (SESSLER, 1994). Pacientes humanos que desenvolvem hipotermia possuem maior taxa de mortalidade do que os que permanecem normotérmicos (JURKOVICH et al., 1987). Esta também exerce efeito inotrópico negativo no miocárdio e a normotermia no período trans-anestésico reduz a incidência de eventos cardíacos mórbidos como isquemia do miocárdio e taquicardia ventricular com alterações cardíacas (FRANK et al., 1997).

A elevação da morbidade vascular pode ser devida, em parte, à elevação dos níveis de norepinefrina, ao acentuado grau de vasoconstrição periférica e ao tônus de receptores $\alpha$-adrenérgicos alterados em resposta ao frio (FRANK et al., 1997). Quando reaquecidos, os pacientes podem apresentar colapso cardiovascular com severa hipóxia tecidual e acidose metabólica (THURMON et al., 1996).

Diversos métodos têm sido utilizados para evitar a perda de temperatura durante o período trans e pós-anestésicos. Em humanos os mais utilizados incluem o aquecimento passivo (temperatura ambiente e cobertores), aquecimento ativo externo (cobertores de ar aquecido, colchão de água circulante aquecida, colchão térmico e aquecedores radioativos) e aquecimento ativo interno (umidificadores, fluidoterapia aquecida e lavagem de cavidade com soluções aquecidas) (SMITH \& YAMAT, 2000).

Muitos desses métodos são de difícil acesso em medicina veterinária devido seu alto custo ou impossibilidade de uso em aves. Destacam-se o colchão térmico, o qual pode causar queimaduras devido à pele fina das aves, a fluidoterapia aquecida, que com uma velocidade de administração lenta impossibilita a manutenção, e os cobertores de ar aquecidos, os quais possuem dimensões inapropriadas para a espécie.

Este estudo objetivou avaliar dois métodos alternativos de manutenção térmica em galinhas domésticas submetidas à anestesia inalatória com isofluorano, utilizando papel alumínio comercial ou manta térmica dupla de alumínio.

\section{MATERIAL E MÉTODOS}

Foram utilizadas seis galinhas (Gallus gallus domesticus), com idade aproximada de um ano e pesando entre 1 e $2 \mathrm{~kg}$. As mesmas foram alojadas em baias recebendo água e ração comercial ad libitum. Os animais foram autocontroles e submetidos a três grupos: alumínio (GA), envoltas por papel alumínio comercial $^{\text {a }}$ com a face refletora (laminada) voltada para o animal; manta (GM), envoltas por manta térmica dupla 
de alumínio $^{\text {b }}$, utilizada como isolante de telhado de residências; controle (GC) em que não foi utilizado nenhum recurso térmico.

Realizado o jejum prévio de 4 horas, os animais foram contidos manualmente em decúbito esternal e a indução anestésica foi realizada por meio de máscara facial com isofluorano ${ }^{\mathrm{a}}$ a $4 \mathrm{~V} \%{ }^{\mathrm{d}}$, sob fluxo

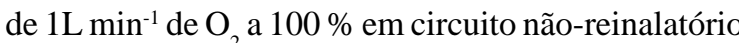
de Bain, com umidificador adaptado. Após a indução, foi realizada intubação com sonda endotraqueal de $4 \mathrm{~mm}$ de diâmetro, sem balonete, e os animais foram posicionados em decúbito lateral esquerdo. A anestesia foi mantida sob vaporização de $2 \mathrm{~V} \%$ de isofluorano.

Os animais foram posicionados sobre um colchão térmico desligado e envoltos em sua totalidade com as mantas térmicas (grupos GA e GM) ou não (GC). Ato contínuo, foram conectados eletrodos para leitura de eletrocardiograma ${ }^{\mathrm{e}}$ e a oximetria ${ }^{\mathrm{f}}$ foi obtida com sensor infravermelho localizado na tíbia e posicionamento de termômetro ${ }^{g}$ via cloacal, permanecendo exposta apenas a cabeça do animal. Os eletrodos foram posicionados nas articulações rádioumero-ulnar e metatarsianas e o sensor de oximetria no metatarso direito. Durante todo o experimento a temperatura da sala cirúrgica foi mantida a $26^{\circ} \mathrm{C}$.

As variáveis mensuradas foram a temperatura corpórea $\left(\mathrm{T}^{\circ}\right)$, em graus Celsius, freqüência cardíaca (FC), em batimentos por minuto, freqüência respiratória (f), em movimentos por minuto e saturação de oxihemoglobina $\left(\mathrm{SatO}_{2}\right)$, em porcentagem, nos momentos zero, cinco, 15, 30, 45, 60, 75 e 90 minutos, sendo que o tempo zero iniciado cinco minutos após a indução anestésica.

A análise estatística foi realizada de acordo com Callegari-Jacques (2003), utilizando-se o programa GraphPad Prism $4^{\mathrm{h}}$. Neste caso, utilizou-se análise de variância (ANOVA) para amostras pareadas, com posterior teste de Dunnett para comparações de médias dentro de cada grupo em relação ao momento zero minuto. Para comparações entre os grupos, em cada momento, foi utilizada ANOVA, seguido de teste de Tukey. As diferenças foram consideradas significantes quando $\mathrm{P}<0,05$. Todos os resultados foram expressos em média \pm desvio padrão.

\section{RESULTADOS}

Observa-se, na tabela 1, que no GC houve hipotermia a partir dos 15 minutos, de aproximadamente $0,5^{\circ} \mathrm{C}$, em que a temperatura inicial foi de $40,9^{\circ} \mathrm{C}$, permanecendo em queda constante até os 90 minutos $\left(-2,1 \pm 0,8^{\circ} \mathrm{C}\right)$ em relação ao momento zero com temperatura final de $38,8^{\circ} \mathrm{C}$. No GA a diferença em relação ao basal $\left(40,9^{\circ} \mathrm{C}\right)$ só ocorreu aos 45 minutos $\left(40,4^{\circ} \mathrm{C}\right)$, com diminuição constante até aos finais dos momentos $\left(-0,9 \pm 0,5^{\circ} \mathrm{C}\right)$ tendo como temperatura final,

Tabela 1 - Variáveis fisiológicas mensuradas em galinhas domésticas submetidas à anestesia inalatória com isofluorano e diferentes sistemas de manutenção térmica.

\begin{tabular}{|c|c|c|c|c|c|c|c|c|}
\hline \multirow{2}{*}{ Parâmetro } & \multirow{2}{*}{ Grupo } & \multicolumn{7}{|c|}{--------------------------------------------------------Minutos------------------------------------------------------ } \\
\hline & & 0 & 15 & 30 & 45 & 60 & 75 & 90 \\
\hline \multirow{3}{*}{$\begin{array}{l}\mathrm{T}^{\circ} \\
\left({ }^{\circ} \mathrm{C}\right)\end{array}$} & Controle & $40,9 \pm 0,8$ & $40,4 \pm 1,0 *$ & $39,9 \pm 1,1^{*}$ & $39,6 \pm 1,3 *$ & $39,3 \pm 1,3 *$ & $39,0 \pm 1,4^{*}$ & $38,8 \pm 1,5 *$ \\
\hline & Alumínio & $40,9 \pm 0,3$ & $40,7 \pm 0,3$ & $40,6 \pm 0,2 \dagger$ & $40,4 \pm 0,3^{*} \dagger$ & $40,3 \pm 0,4 * \dagger$ & $40,1 \pm 0,4^{* \dagger}$ & $40,0 \pm 0,5^{* \dagger}$ \\
\hline & Manta & $41,0 \pm 0,7$ & $40,7 \pm 0,6^{*}$ & $40,4 \pm 0,7^{*}$ & $40,3 \pm 0,6 * \dagger$ & $40,2 \pm 0,7 * \dagger$ & $40,0 \pm 0,7 * \dagger$ & $39,9 \pm 0,7 * \dagger$ \\
\hline \multirow{3}{*}{$\begin{array}{l}\text { FC } \\
\text { (bpm) }\end{array}$} & Controle & $205 \pm 17$ & $183 \pm 24 *$ & $171 \pm 25^{*}$ & $163 \pm 28 *$ & $158 \pm 25^{*}$ & $149 \pm 24^{*}$ & $147 \pm 24^{*}$ \\
\hline & Alumínio & $203 \pm 23$ & $196 \pm 33$ & $189 \pm 31$ & $186 \pm 28$ & $177 \pm 28^{*}$ & $176 \pm 33^{*}$ & $177 \pm 40^{*}$ \\
\hline & Manta & $208 \pm 12$ & $204 \pm 16$ & $194 \pm 19 *$ & $189 \pm 20 *$ & $185 \pm 22 *$ & $177 \pm 19 *$ & $169 \pm 16^{*}$ \\
\hline \multirow{3}{*}{$\begin{array}{l}f \\
(\mathrm{mpm})\end{array}$} & Controle & $7 \pm 2$ & $6 \pm 2$ & $6 \pm 2$ & $5 \pm 2 *$ & $5 \pm 2 *$ & $5 \pm 2 *$ & $5 \pm 2 *$ \\
\hline & Alumínio & $6 \pm 2$ & $5 \pm 1 *$ & $4 \pm 1^{*}$ & $4 \pm 1 *$ & $4 \pm 1 *$ & $4 \pm 1^{*}$ & $4 \pm 1^{*}$ \\
\hline & Manta & $8 \pm 3$ & $8 \pm 4$ & $7 \pm 4 *$ & $7 \pm 4 *$ & $7 \pm 4 *$ & $6 \pm 4^{*}$ & $6 \pm 3^{*}$ \\
\hline \multirow{3}{*}{$\begin{array}{l}\mathrm{SatO}_{2} \\
(\%)\end{array}$} & Controle & $97 \pm 1,4$ & $97 \pm 1,1$ & $97 \pm 1,3$ & $97 \pm 1,3$ & $97 \pm 1,3$ & $98 \pm 1,2$ & $98 \pm 1,0$ \\
\hline & Alumínio & $97 \pm 0,6$ & $97 \pm 0,6$ & $97 \pm 0,6$ & $97 \pm 0,6$ & $97 \pm 0,5$ & $97 \pm 0,0$ & $97 \pm 0,5$ \\
\hline & Manta & $96 \pm 1,5$ & $97 \pm 1,7$ & $97 \pm 0,5$ & $97 \pm 0,8$ & $97 \pm 0,5$ & $97 \pm 0,8$ & $97 \pm 0,8$ \\
\hline
\end{tabular}

* Diferença significante em relação ao tempo 0 (Dunnett, $\mathrm{P}<0,05$ ).

$\dagger$ Diferença significante em relação ao grupo controle (Tukey, $\mathrm{P}<0,05$ ).

$\mathrm{T}^{\circ}=$ temperatura; $\mathrm{FC}=$ freqüência cardíaca; $f=$ freqüência respiratória; $\mathrm{SatO}_{2}=$ Saturação de oxihemoglobina

Ciência Rural, v.38, n.5, ago, 2008. 
$40^{\circ} \mathrm{C}$. No GM houve hipotermia significativa com 15 minutos $\left(40,7^{\circ} \mathrm{C}\right)$ em relação ao basal $\left(41^{\circ} \mathrm{C}\right)$, permanecendo em queda; aos 90 minutos a perda foi de $1,1\left( \pm 0,2^{\circ} \mathrm{C}\right)$ com valor final de $39,9^{\circ} \mathrm{C}$. Comparandose os grupos, a temperatura foi significativamente maior aos 30 minutos $\left(40,6^{\circ} \mathrm{C}\right)$ no GA e aos 45 minutos $\left(40,3^{\circ} \mathrm{C}\right)$ no GM, em relação ao GC.

Os animais apresentaram bradicardia em

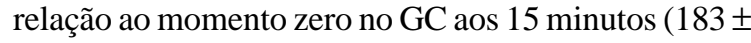
24), no GM aos 30 minutos (194 \pm 19 ) e no GA apenas após 60 minutos (177 \pm 28$)$. Entretanto, não se observou diferença significativa entre os grupos.

Nos três grupos a $f$ só apresentou diferença significativa em relação ao momento zero, não diferindo entre os mesmos. Esta variação ocorreu aos 15 minutos (5 \pm 1$)$ no GA, aos 30 minutos no GM (7 \pm 4$)$ e aos 45 minutos no GC $(5 \pm 2)$. A saturação de oxihemoglobina não apresentou nenhuma alteração significativa.

\section{DISCUSSÃO}

A hipotermia em aves no período transanestésico pode interferir negativamente com o retorno ao estado fisiológico normal, promovendo respostas fisiológicas alteradas, reduzindo a eficiência do sistema cardiovascular, a atividade cerebral e a função hepática excretora (REMBERT et al., 2001), ocasionando óbitos (BOEDEKER et al., 2005). Também pode ocorrer óbito pela depressão do centro respiratório (MUIR \& HUBBELL, 2001), tornando a monitoração da temperatura corpórea de extrema importância, sendo recomendado por KORBEL (1998) a temperatura acima de $40^{\circ} \mathrm{C}$.

Durante a anestesia, há diminuição da produção de calor pelo decréscimo da função metabólica e atividade muscular (REMBERT et al., 2001; BOEDEKER et al., 2005), além da ação dos agentes anestésicos inalatórios na inibição da insuflação da plumagem (CARTER-STORM, 1987) e diminuição da resistência vascular sistêmica (PRECKEL \& BOLTEN, 2005).

Como visto anteriormente, a hipotermia pode deprimir as funções orgânicas, tanto bioquímica quanto fisiologicamente, não sendo surpresa que as respostas aos fármacos sejam exacerbadas durante a hipotermia (WONG, 1983). Isso pode explicar o decréscimo constante nos valores dos parâmetros avaliados, devido à potencialização dos fármacos ocasionada pela redução na temperatura.

Observou-se maior hipotermia no GC, o que pode ser explicada pela maior troca de calor do animal com o meio ambiente devido à vasodilatação periférica, e também por meio da inabilidade da insuflação da plumagem. GUIMARÃES (2000) relatou que a hipotermia em galinhas foi maior no grupo anestesiado com isofluorano, devido à ocorrência de maior hipotensão, quando comparada com o halotano e sevofluorano, permitindo assim, maior perda de calor para o ambiente devido à vasodilatação periférica. Por serem isolantes térmicos, o alumínio e a manta evitaram maior perda de calor do animal com o ambiente.

No presente estudo, pode-se sugerir que o alumínio, por ter uma espessura fina e possuir uma face refletora, necessita de um período menor para ser aquecido, evitando assim uma maior perda de temperatura, ao contrário da manta que, sendo mais espessa, necessita de um período maior, resultando em maior perda de temperatura pelo animal.

A utilização de um método de aquecimento ativo interno, no caso o umidificador, pode ter contribuído para uma melhor manutenção da temperatura corpórea, corroborando Dodman \& BritoBabapulle (1979), apud HALL et al. (2001), os quais enfatizaram que o uso de um umidificador pode evitar maiores perdas na temperatura devido à umidificação dos gases inspirados. Segundo KORBEL (1998), o fornecimento de calor por meio de fontes externas isoladamente não é completamente eficaz devido à ação isolante da plumagem.

Em estudo realizado por GOELZ et al. (1990), observou-se taquicardia em patos de Pequim anestesiados com 2 a 3V\% de isofluorano, resultado não observado no presente estudo. GREENLES et al. (1990) observaram bradicardia e hipotensão em galinhas da raça Broiler mantidas sob anestesia inalatória com isofluorano (2,1V\%) e halotano (1,2V\%), sugerindo que os efeitos depressores foram devido à ação direta dos fármacos no sistema cardiovascular, tendo em vista que os animais estiveram sob ventilação controlada.

No presente estudo, houve bradicardia em todos os grupos em relação ao basal, fato que pode ser explicado pela utilização do agente anestésico, o qual inibe o influxo de íons cálcio nas células do miocárdio, promovendo efeito inotrópico negativo (PRECKEL \& BOLTEN, 2005). A hipotermia é outro fator que pode ter contribuído para bradicardia, exacerbando esse efeito inotrópico negativo (SMITH \& YAMAT, 2000).

De acordo com PRECKEL \& BOLTEN (2005), a anestesia inalatória promove taquipnéia compensatória à queda do volume corrente. Apesar de não ter sido avaliado o volume corrente, observou-se bradipnéia neste estudo, em todos os grupos, o que corrobora PAULIN (1986), o qual diz que os agentes inalatórios deprimem a freqüência respiratória de forma significativa em pacientes sob ventilação espontânea. Isso decorre da dessensibilização dos receptores 
intrapulmonares para as alterações nos níveis de $\mathrm{CO}_{2}$ nas aves com o uso dos anestésicos inalatórios (PIZARRO et al., 1990). Comparando-se os principais agentes, o isofluorano é o que promove maior depressão respiratória entre os anestésicos halogenados (PAULIN, 1986; STEFFEY, 1996).

\section{CONCLUSÃO}

Ambos os métodos empregados foram eficientes na manutenção térmica, sendo que o alumínio foi mais eficiente devido a menor variação de temperatura e de freqüência cardíaca, devido a menor espessura e maior maleabilidade, evitando assim maior contato com o ar ambiente. Nesse caso, tem-se uma opção de manutenção térmica eficiente, de fácil aquisição e baixo custo.

\section{FONTES DE AQUISIÇÃO}

aPapel Alumíno Conserv, Conserv Indústria de Embalagens, Porto Alegre, RS.

bPapéis Gomados Líder e Conexos S/A, São Bernardo do Campo, SP.

'Isoforine, Cristália Produtos Químicos e Farmacêuticos Ltda. Itapira, SP.

${ }^{d}$ HB Hospitalar Indústria e Comércio Ltda, São Paulo, SP.

'ECGPC TEB, TEB Tecnologia Eletrônica Brasileira Ltda. São

Paulo, SP.

fSolo DX1265 Dixtal Biomédica Ind. e Com. Ltda. São Paulo, SP.

BDD, Becton Dickinson Brasil, São Paulo, SP.

${ }^{\text {h} G r a p h P a d ~ P r i s m, ~ G r a p h P a d ~ S o f t w a r e ~ I n c, ~ S a n ~ D i e g o, ~ C A, ~ U S A . ~}$

\section{COMITÊ DE ÉTICA E BEM-ESTAR ANIMAL}

O artigo foi aprovado pelo Comitê de Ética e Bem-Estar Animal da Universidade Federal de Santa Maria, com número de protocolo 40/2007.

\section{REFERÊNCIAS}

BOEDEKER, N.C. et al. Comparison of body temperature of pigeons (Columbia livia) anesthetized by three different anesthetic delivery systems. Journal of Avian Medicine and Surgery, v.19, n.1, p.1-6, 2005.

CALLEGARI-JACQUES, S.M. Bioestatística: princípios e aplicações. Porto Alegre: Artmed, 2003. 255p.

CARTER-STORM, A. Special considerations for general anesthesia of birds. Modern Veterinary Practice, v.68, n.6, p.358-360, 1987.

CURRO, T.G. et al. Determination of the ED50 of isoflurane ane evaluation of the isoflurane-sparing effects of butorphanol in Cockatoos (Cacatua spp.). Veterinary Surgery, v.23, p.429-433, 1994.

FRANK, S.M. et al. Perioperative maintenance of normothermia reduces de incidence of morbid cardiac events: a randomized clinical trial. Journal of the American Medical Association, v.227, p.1127-1134, 1997.

FLECKNELL, P.A. Anaesthetic management. In: Laboratory animal anaesthesia. London: Academic, 1996. p.75-100.

GOELZ, M.F. et al. Effects of halothane and isoflurane on mean arterial blood pressure, heart rate, and respiratory rate in adult Pekin ducks. American Journal of Veterinary Research, v.51, n.3, p.458-460, 1990

GREENLESS, K.J. et al. Effect of halothane, isoflurane, and pentobarbital anesthesia on myocardial irritability in chickes. American Journal of Veterinary Research, v.51, n.5, p.757-758, 1990.

GUIMARÃES, L.D. Estudo comparativo entre sevofluorano, halotano e isofluorano em Gallus Domesticus. Ciência Rural, v.30, n.6, p.999-1004, 2000 .

GUNKEL, C.; LAFORTUNE, M. Current techniques in avian anesthesia. Seminars in Avian and Exotic Pet Medicine, v.14, n.4, p.263-276, 2005.

HALL, L.W. et al. Prevention and management of anaesthetic accidents and crises. In: London: Saunders, 2001. p.507-533.

JURKOVICH, G.J. et al. Hypothermia in trauma victims: an ominous predictor of survical. Journal of Trauma, v.27, p.1019-1024, 1987.

KORBEL, R. Vergleichende untersuchungen zur inhalationsanaästhesie mit isofluran (Forene ${ }^{\circledR}$ ) und sevofluran (SEVOrane $^{\circledR}$ ) bei haustauben (Columba livia gmel., 1789, var. domestica) und vorstellung eines referenz - narkoseprotokolls für vögel. Tierarztliche Práxis, v.26, p.211-223, 1998.

LUDDERS, J.W. et al. Minimun anesthetic dose and cardiopulmonary dose response for halothane in chickens. American Journal of Veterinary Research, v.49, n.6, p.929-932, 1988.

LUDDERS, J.W. Minimal anesthetic concentration and cardiopulmonary dose-response of halothane in ducks. Veterinary Surgery, v.21, n.4, p.319-324, 1992.

MUIR III, W.W.; HUBBELL, J.A.E. Procedimentos anestésicos em animais de estimação exóticos. In: MUIR III, W.W. et al. Manual de anestesia veterinária. 3.ed. Porto Alegre: Artmed, 2001. p.297-323.

NAGANOBU, K. et al. Arrhythmogenic effect of hypercapnia in ducks anesthetized with halothane. American Journal of Veterinary Research, v.62, n.1, p.127-129, 2001.

OLKOWSKI, A.A.; CLASSEN, H.L. Safety of isoflurane anaesthesia in high risk avian patients. Veterinary Record, v.143, p.82-83, 1998 .

PAULIN, E.G. Respiratory pharmacology of inhalated anesthetic agents. In: MILLER, R.D. Anesthesia. 2.ed. New York: Chunchill Livingstone, 1986. p.667-699.

PASCOE, P.J. et al. Avian anaesthesia. Veterinary Record, v.116, n.2, p.58, 1985. 
PIZARRO, J. et al. Halothane effects on ventilatory responses to changes in intrapulmonary $\mathrm{CO}_{2}$ in geese. Respiratory Physiology, v.82, p.337-348, 1990.

PRECKEL, B.; BOLTEN, J. Pharmacology of modern volatile anaesthetics. Best Practice \& Research Clinical Anesthesiology, v.19, n.3, p.331-348, 2005.

REMBERT, M.S. et al. Comparison of traditional thermal support devices with the forced-air warmer system in anesthetized Hispaniolan Amazon Parrots (Amazona ventralis). Journal of Avian Medicine and Surgery, v.15, n.3, p.187193, 2001.

SESSLER, D.L. Consequences and treatment of perioperative hypothermia. Anesthesiology Clinics of North America, v.12, p.425-456, 1994.
SMITH, C.E.; Yamat, R.A. Avoiding hypothermia in the trauma patient. Current Opinion in Anaesthesiology, v.13, p.167$174,2000$.

STEFFEY, E. Inhalation anesthetics. In: THURMON, J.C. et al. Lumb \& Jones' veterinary anesthesia. 3.ed. Baltimore: Williams \& Wilkins, 1996. p.297-304.

THURMON, J.C. et al. Injectable anesthetics. In: Lumb \& Jones' veterinary anesthesia. 3.ed. Baltimore: Willians \& Wilkins, 1996. p.210-240.

WATERMAN, A.E. Maintenance of body temperature during anaesthesia. Proceedings of Association of Veterinary Anaesthetists of Great Britain and Ireland, v.9, p.73-85, 1981.

WONG, K.C. Physiology and pharmacology of hypothermia. Western Journal of Medicine, v.138, n.2, p.227-232, 1983. 